Research Article

\title{
A Computational Simulation Technology for Specific Counting of Perinatal and Postnatal Human Tissue Stem Cells for Transplantation Medicine
}

Renly Dutton ${ }^{1, \neq}$, Frank Abdi $^{1}$, Levon Minnetyan ${ }^{1}$, James L. Sherley ${ }^{2, *}$

1. AlphaSTAR Corporation, Long Beach, CA, 90804, USA; E-Mails: renly.dutton@gmail.com; fabdi@alphastarcorp.com; levon@clarkson.edu

2. Asymmetrex, LLC, P.O. Box 301179, Boston, MA, 02130-1822, USA; E-Mail: jsherley@asymmetrex.com

¥ Current Address: 402A Flora Lane, Scotts Valley, CA 95066, USA; E-Mail: renly.dutton@gmail.com

* Correspondence: James L. Sherley; E-Mail: jsherley@asymmetrex.com

Academic Editor: Robert C. McCarthy

Special Issue: Isolation and Characterization of Adult Therapeutic Cells

OBM Transplantation

2020, volume 4, issue 3

doi:10.21926/obm.transplant.2003117
Received: April 22, 2020

Accepted: August 20, 2020

Published: August 28, 2020

\begin{abstract}
Although tissue stem cells are essential for the maintenance, renewal, and repair of vertebrate organs and tissues, previously, the simple act of counting them has not been possible. For more than a half-century, progress in tissue stem cell research and medicine has been undermined by the lack of a means to determine tissue stem cell number. In particular, a major unmet need for stem cell transplantation medicine has been a way to quantify the specific dosage of tissue stem cell treatments. The counting problem persists because no biomarkers are known that identify tissue stem cells specifically, without also counting their more abundant committed progenitor progeny cells. Here, we describe integration of principles of tissue stem cell asymmetric self-renewal kinetics with computational simulation to achieve specific and accurate counting of therapeutic tissue
\end{abstract}

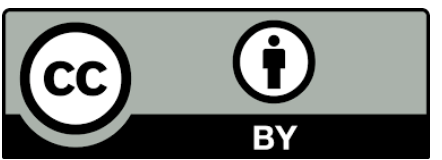

(C) 2020 by the author. This is an open access article distributed under the conditions of the Creative Commons by Attribution License, which permits unrestricted use, distribution, and reproduction in any medium or format, provided the original work is correctly cited. 
stem cells. The asymmetric self-renewal kinetics of tissue stem cells is rate-limiting for the rate and extent of proliferation of primary tissue cell cultures. Based on this essential relationship, we show that simple total cell count data from primary cell cultures, passaged until achieving terminal proliferation arrest, are determined by tissue stem cell kinetics factors, including viability, cell cycle time, self-renewal rate, and number. We describe a Probabilistic Stem Cell Kinetics (PSCK) model and Random Input Factor Searching (RIFS) software that can be combined to discover these previously inaccessible factors. The method is validated by several orthogonal strategies, including comparisons to the currently best available method for estimating a tissue stem cell fraction, independent tests for asymmetrically self-renewing cells, tissue stem cell fractionation, and treatments with tissue stem cell-active agents. A major advance of the method is the discovery of simple algorithms that allow rapid convenient computation of the specific tissue stem cell fraction of complex human tissue cell preparations from simple population doubling time data.

\section{Keywords}

Tissue stem cells; transplantation medicine; stem cell transplantation; asymmetric selfrenewal; cumulative population doublings; cell kinetics; computer simulation; hematopoietic stem cells; cord blood; mesenchymal stem cells

\section{Introduction}

Though often overlooked and poorly understood, a stem cell counting problem for perinatal and postnatal vertebrate tissues, including those of humans, has persisted for more than a halfcentury [1-3]. Two biological properties of adult tissue stem cells (i.e., perinatal and postnatal tissue stem cells) are mainly responsible for this situation. First, because stem cells exist in low fractions in tissues, they also have low fractions in isolated tissue cell preparations. This tissue property is often unappreciated because of the common misconception and misrepresentation that stem cell-containing preparations are homogenous for stem cells. In fact, the most enriched populations of adult tissue stem cells rarely exceed fractions greater than a few percent. Second, commonly misnamed "stem cell biomarkers" used for tissue stem cell research are also expressed by much more abundant committed progenitor cells [1-3]. As the progeny of asymmetrically selfrenewing stem cell divisions, based on their molecular expression properties defined to date, committed progenitors have been indistinguishable from their stem cell parents. Since committed progenitor cells outnumber stem cells by orders of magnitude both in vivo and in isolated tissue cell preparations, present day tissue "stem cell biomarkers" actually give only a committed progenitor cell count, and no stem cell count at all.

Despite the long-standing unmet need for methods for specific and accurate counting of perinatal and postnatal tissue stem cells, there is significant misinformation on this issue in stem cell research and stem cell medicine. Though not all, many scientists, physicians, and technologists operate with the misperception that tissue stem cell counting is possible and that the stem cellspecific dosage of treatments is known. The basis for this confusion is two-fold, being related to the language of stem cell science and medicine and to the limitations of the available methods 
that attempt to quantify tissue stem cells. First, the language of tissue stem cell quantification is often not sufficiently exact. Recent controversy over the constituents of mesenchymal stem cell ("MSC")-containing tissue cell preparations and the naming of the preparations is illustrative of this problem [4]. MSC preparations are not homogeneous collections of tissue stem cells. Like all isolated, enriched, or expanded tissue cell preparations, they are heterogeneous. They contain mesenchymal stem cells, lineage-related mesenchymal committed progenitor cells, and both lineage-related and non-lineage mature cells. The mesenchymal stem cell fraction is a small proportion of total cells. Low stem cell fraction is characteristic of all types of stem cell-containing tissue cell preparations, including enriched populations like CD34+-selected cell fractions containing hematopoietic stem cells (HSCs). When correct exact language is used, tissue stem cellcontaining preparations are called "stem/progenitor cells." However, often used less exact language, like "stem cells" per se, is routinely applied inappropriately and fosters the mistaken idea that designated stem cell preparations are homogenous for stem cells, when in fact they never are. This error in characterization leads to the misperception that simple counts of the total cell number are equivalent to a stem cell counts and stem cell dose determination, though they are not. Some investigators working with "MSCs" have suggested renaming the preparations to acknowledge the uncertainty of cellular constituents [4]. However, their solution, though more exact, obscures the significance of the crucial cellular constituent, the stem cell fraction. A means to provide the stem cell-specific fraction of MSC preparations is a better solution all around.

A second cause of significant misinformation and confusion about the state of tissue stem cell quantification methods is misunderstanding of the capabilities of existing methods for estimating tissue stem cell number. A very common belief is that tissue stem cells can be counted by flow cytometry. However, although antibodies exist that allow tissue stem cells to be enriched by either fluorescent activated cell sorting (FACS) or immunoselection, the same antibodies cannot be used to count tissue stem cells by flow cytometry. As noted earlier, all known molecular biomarkers expressed by tissue stem cells are also expressed by their committed progenitor cell progeny [1-3]. Since, committed progenitor cells outnumber stem cells significantly both in vivo and in isolated stem cell-enriched cell populations, but appear the same, they prevent quantification of the smaller fraction of tissue stem cells, preventing the latter's quantification by flow cytometry.

Despite the fact that previously no method existed to count human tissue stem cells specifically and accurately, confusion and misinformation in tissue stem cell science and medicine about the issue of tissue stem cell quantification has been persistent. There are several misnamed "stem cell biomarkers" that are commonly mistaken for being able to quantify tissue stem cells. Examples of misrepresented biomarkers are CD34, CD133, and CD90 [1-3]. These molecular biomarkers are proteins expressed on respective types of tissue stem cells. As noted before, their oftenunappreciated shortcoming is that they are also expressed on the differentiated progeny cells produced by asymmetrically self-renewing tissue stem cells. These committed progenitor cells do not have the therapeutically sought durable, long term, tissue cell renewing properties of tissue stem cells, but they express the same so-called "stem cell biomarkers." In freshly isolated tissue cell preparations, and even more so in cultured tissue cell preparations produced in cell biomanufacturing [5], committed progenitor cells can outnumber the tissue stem cells by 100 -fold or more. Therefore, counts made with these, the currently best available molecular biomarkers, are not tissue stem cell counts, but instead committed progenitor cell counts [1]. 
There are two other main assays in wide usage that are often mistaken for being able to determine the fraction of tissue stem cells. The colony forming unit (CFU) assay is prevalent in the cord blood banking industry for evaluating the hematopoietic stem/progenitor cell fraction; and it is also used for estimating endothelial stem/progenitor cell number. Both applications suffer from the well known inability of the CFU assay to distinguish colonies formed by stem cells from colonies formed by early committed progenitor cells. This lack of specificity is the long recognized cause of the CFU assay's failure to predict the HSC transplantation potency of umbilical cord blood units [6].

Unlike the CFU assay, the limiting-dilution SCID mouse repopulating cell (LDSRC) assay has superior specificity for detection of the activity of human HSCs, from adult donors or umbilical cord blood. Currently, gene therapy companies whose therapies target human HSCs use this assay to estimate the HSC fractions at different stages of their therapeutic development process. Despite its excellent specificity, the LDSRC assay has several significant drawbacks. Because of its requirement of a large number of mice to perform, it is very expensive. Requiring 8-12 weeks to complete, it lacks the timeliness needed for the process engineering of HSC biomanufacturing and dosage determination for stem cell treatments. Though it detects stem cells specifically, its quantification accuracy is limited by variability in the engraftment efficiency of the tester mice. Finally, it is only applicable to HSCs and not stem cells from other tissues [7].

In addition to the CFU assay and the LDSRC assay, there are other cell assays in the commercial tissue stem cell marketplace that quantify particular cellular metabolic activities that are also mistaken to be tissue stem cell-specific [8]. Therefore, despite their representation, none of these assays quantify tissue stem cells. In addition, unlike the new tissue stem cell counting method described in this report, none of the other currently available methods for attempting to estimate tissue stem cell fraction also provide determination of tissue stem cell-specific kinetics factors for viability, cell cycle time, and symmetric self-renewal rate. Each of these tissue stem cell-specific properties could become important new metrics and tools for accelerating progress in tissue stem cell science and medicine.

There is one property of adult tissue stem cells that does distinguish them from committed progenitor cells. This property is their asymmetric self-renewal kinetics $[9,10]$. Asymmetric selfrenewal is the process by which adult tissue stem cells continuously divide with simultaneous renewal of themselves and production of lineage-committed progenitor cells. Herein, we describe the design and validation of a first method for specific and accurate counting of stem cells from diverse human tissues based on the concept that asymmetric self-renewal by adult tissue stem cells defines them exclusively and is rate-determining for the overall proliferation of primary human cell preparations. The new counting technology addresses many previously unmet needs in tissue stem cell research, tissue stem cell transplantation medicine, drug development, and toxicology. It can determine, for the first time, the number of tissue stem cells in experimental cultures, the dose and quality of therapeutic stem cell preparations, and positive or negative effects of agents on tissue stem cell function specifically.

To determine the stem cell-specific fraction of human tissue cell preparations, the presented approach integrates computer simulation with a bioengineered model of the cell kinetics of total cell production that depends on the asymmetric self-renewal of tissue stem cells present in cultures. This bioengineered computational approach yields important, previously inaccessible, 
tissue stem cell kinetics factors, in addition to the stem cell-specific fraction. The new method quantitatively differentiates stem cells from committed progenitor cells for the first time.

To validate the new tissue stem cell counting method, several different orthogonal validation bases are presented. Since there is no existing method for counting tissue stem cells, validation was based on both comparisons to the best available estimates of a tissue stem cell fraction and predicted changes in stem cell fraction after experimental manipulation. Published estimates of human HSC fraction, determined by LDSRC assays, were used as best available estimates of a tissue stem cell fraction for comparison. Comparisons were also made to published determinations of the fraction of asymmetrically self-renewing cells, because this is the primary factor quantified by the counting method. In addition, validation was obtained by confirming changes in tissue stem cell fraction determinations in response to treatment of primary cell cultures with agents either known or suspected to cause changes in tissue stem cell self-renewal or viability. In the case of stem cell-toxic agents, these validation analyses also provided the first demonstration that a chemotherapy agent had specific toxicity against tissue stem cells compared to committed progenitor cells.

As a computational approach, the new method also provides, for the first time, the opportunity to relate changes in tissue stem cell fraction directly to changes in the population doubling time of primary human cell cultures. Such unique analyses reveal mathematical algorithms that now allow the rapid and convenient determinations of the stem cell fraction of complex human tissue cell cultures requiring only simple, readily available cell culture population doubling time data. We anticipate that the advances described in this report will close the chapter on adult tissue stem cells as elusive, unquantifiable elements of vertebrate tissues; and open a new chapter of accelerated progress in stem cell research, stem cell transplantation medicine, and drug discovery.

\section{Materials and Methods}

\section{$2.1 \mathrm{Cells}$}

Strain WI-38 pre-senescent human lung cells were purchased from the American Type Culture Collection (Manassas, VA, USA; ATCC \#CCL-75; passage 15). Expanded human liver stem cell strain SACK-XS 12(3) was purchased from Kerafast (Boston, MA, USA; \#EJS002). CD34+-selected normal human bone marrow cells were purchased from the ATCC (\#PCS-800-012). CD34+-selected human umbilical cord blood cells were purchased from STEMCELL Technologies, Inc. (Cat\# 70008; Cambridge, MA).

After the completion of the described analyses, the authors became aware that strain WI-38 cells were derived from the tissues of an electively aborted human fetus [11]. As a result of this awareness, strain WI-38 cells will not be used in future studies by the authors. Discontinuation of the use of these cells is also recommended for future studies by others.

\subsection{Cell Culture}

Serial cultures of WI-38 cells were performed in Dulbecco's Minimal Essential Medium (DMEM) supplemented with $10 \%$ dialyzed fetal bovine serum (dFBS) by the University of Maryland Biotechnology Scale-up Facility (College Park, MD, USA) per the authors' specifications. Starting with an input of 65,000 viable (i.e., trypan blue-negative) cells in $5 \mathrm{mLs}$ medium in wells of 6-well 
culture plates, six replicate cultures were serially passaged every $96 \pm 4$ hours by transferring $1 / 3$ of the total cells to a new culture well. Triplicate total and viable cell counts were performed for each serial culture at each passage. Passaging was continued until two successive cell counts showed no significant increase in total cell number above the input cell number for the culture.

Culture analyses for liver stem cells and bone marrow-derived stem cells were performed by Toxikon, Inc. (Bedford, MA, USA) per the authors' specifications. Expanded human liver stem cell strain SACK-XS 12(3) cells were cultured in the supplier's recommended culture medium without inclusion of xanthosine. CD34+-selected normal human bone marrow cells were cultured in StemSpan $^{\text {TM }}$ SFEM II medium (STEMCELL Technologies, Inc., Cambridge, MA, USA; \#09655) supplemented with StemSpan ${ }^{\mathrm{TM}}$ CD34+ Expansion Supplement (STEMCELL Technologies, Inc., \#02691). SACK-XS 12(3) serial cultures were initiated in 6-well plates with 325,000 viable cells. CD34+ cell serial cultures were initiated as 24 -well suspension cultures with 65,000 viable cells. For both cell types, $1 / 3$ of total cells were transferred to a new culture well every $96 \pm 4$ hour interval. Tested agents were supplemented at all times during the culture period. Passaging was continued until successive total and viable cell counts showed no significant increase in total cell number above the transferred cell number.

The serial cell culture and viable cell count determinations for CD34+-selected human umbilical cord blood cells were performed by the Cell Culture Core Facility at University of MassachusettsAmherst (Amherst, MA) per procedures provided by the authors. Serial 24-well suspension cultures were initiated with 177,000 total cells in StemSpan ${ }^{\text {TM }}$ SFEM II culture medium (Stemcell Technologies, Cat\# 09655) supplemented with StemSpan ${ }^{\mathrm{TM}}$ CD34+ Expansion Supplement (Stemcell Technologies, Cat\# 02691) and 1\% penicillin/streptomycin. Triplicate suspension cultures were passaged with 1:5 splits every $72 \pm 1.4$ hours until two successive cell counts showed no significant increase in total cell number above the transferred cell number.

All other culture cell count data were obtained from the referenced published reports.

\subsection{Correction Analysis for Initial Stem Cell Fraction}

PSCK-RIFS analyses revealed that some human tissue stem cells have a measurable, significant rate of symmetric self-renewal during cell culture. Because the resolution of the PSCK-RIFS determination of the initial stem cell fraction of input cells is limited by the size of the initial passage interval (3-4 days), cultures that have a significant degree of symmetric self-renewal will yield an initial stem cell number ( $\mathrm{NS}_{0}$ below) that is higher than the actual value before culture. Based on the determined stem cell symmetric self-renewal frequency (RS; See Additional Materials Table S1), we apply a correction to account for this effect. The correction is determined by the following Equation 1:

Eq 1 NS $_{\text {o }}$ corrected $=\mathbf{N S}_{1 \text { st interval }} /\left(\mathrm{RSe}^{\mathrm{kt}}+\mathrm{RA}-\mathrm{RDS}\right)$, where...

$\mathrm{NS}_{1 \text { st interval }}=\mathrm{NS}$ peak value determined from the initial interval of PSCK. $\mathrm{t}=$ the time to the first interval NS peak in the respective simulation. $k=\ln 2 / G T S$, the mean generation time for symmetrically self-renewing stem cells. RS, RA, and RDS are mean cell kinetics factors determined by the originating PSCK-RIFS analysis (See Additional Materials Table S1). The mean of 10 independent PSCK NS simulations was used for correction. Eq 1 was applied to determine the initial stem cell fraction whenever a significant RS value was found. 


\subsection{Statistical Analyses}

The simulation confidence level was estimated from the root mean squared error of the comparison of simulated cumulative population doubling (CPD) data to experimental CPD data. Student's t-test (2-tailed) was used to calculate $p$-values for confidence for cell kinetics factors determined by the PSCK-RIFS software with respect to 0.0 and their $95 \%$ confidence intervals. Student's t-test (2-tailed) was also used to calculate $p$-values for confidence for differences in the value of cell kinetics factors derived under different culture conditions.

\section{Results}

\subsection{The Cellular Basis for a Probabilistic Stem Cell Kinetics (PSCK) Model for the Production of Normal Tissue Cells in Culture}

We considered that the unique asymmetric self-renewal of adult tissue stem cells could be used to quantify them. In vivo, tissue stem cells are postulated to divide with asymmetric selfrenewal kinetics $[10,12,13]$. By this cell kinetics program, stem cells can divide to produce cells committed to differentiation while maintaining their own number and stem cell properties. Illustrated in Figure 1, this stem cell kinetics model includes both deterministic and probabilistic features. An essential probabilistic feature is the likelihood that the stem cell divides symmetrically versus asymmetrically. Symmetric divisions produce stem cell duplication, which increases the stem cell tissue fraction [14]; whereas asymmetric divisions maintain and renew tissue cells while preserving stem cell number $[10,12,13]$.

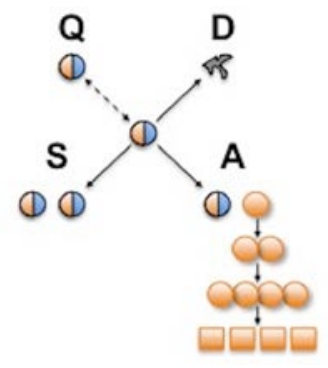

Figure $1 \mathrm{~A}$ probabilistic stem cell kinetics (PSCK) cellular model for cell production by adult tissue stem cells in culture based on tissue stem cell asymmetric self-renewal. In cell culture, adult tissue stem cells (bivalent circles) exist in one of four probabilistic cell kinetics states that are postulated to be extensions of analogous functions in tissues $[10,12,13] . Q$, quiescence, the state of reversible cell division arrest. $D$, the state of cell death. $\mathrm{S}$, the state of symmetric self-renewal that produces two stem cells. A, the state of asymmetric self-renewal, which maintains a constant level of tissue stem cells while simultaneously producing committed progenitor cells (solid circles) that produce differentiated cell lineages that often end with terminally-arrested, nondividing mature cells (squares). Generally, in tissues in vivo and in cell culture ex vivo, the probability of the A state is significantly greater than the probability of the S state. In vivo, this relationship maintains homeostatic tissue cell kinetics. Ex vivo during serial cell culture, the same relationship is predicted to cause a reduction in tissue stem cell fraction by cellular dilution [14-17]. 
Asymmetric cell kinetics by tissue stem cells can explain the characteristic eventual growth cessation of pre-crisis and pre-senescent serial cell cultures $[15,16]$. Another hypothesis proposed to explain these respective rodent and human cell division arrests is telomere erosion. However, one feature of crises and senescence is not accounted for by telomere erosion. Both are cell density-dependent processes $[18,19]$. Serial passage at higher cell densities delays the cell division arrest of cultures. This phenomenon can be explained by asymmetric self-renewal kinetics, which are highly density-dependent, with symmetric self-renewal being more probable at higher cell densities [14, 15].

In the absence of mutations that disrupt asymmetric self-renewal kinetics, cell cultures derived from diverse mammalian tissues undergo total division cessation after serial passage at sufficiently low cell dilutions. Called crisis for rodent cell cultures, the division cessation is often followed by a later recovery of cell division, an event called immortalization [20]. In contrast, the senescence arrest of all human tissue cultures is terminal, with one described exception. Fibroblasts from Li-Fraumeni Syndrome patients, who have a heterozygous p53 gene mutation, do undergo immortalization, but with loss or mutation of the wild-type p53 allele [21]. In a related fashion, immortalized rodent cell lines invariably have acquired p53 gene mutations [22] and lost the ability to undergo asymmetric self-renewal kinetics $[15,23]$. Moreover, restoration of wildtype $\mathrm{p} 53$ function restores asymmetric self-renewal kinetics [15, 23].

Based on our earlier observations of p53 effects on asymmetric stem cell kinetics, we proposed that the total cell output of serial mammalian tissue cell cultures is related to the fraction and selfrenewal pattern of their tissue stem cells [15]. When the frequency of asymmetric self-renewal divisions by stem cells exceeds the frequency of symmetric self-renewal divisions, serial passaging will cause the loss of tissue stem cells as a result of their dilution among their differentiated progeny cells $[5,14-16]$. Therefore, "crisis" or "senescence" as described are primarily states of cell division arrest due to the absence of stem cells. The number of passages required to achieve cell culture division arrest is related to factors that describe the cell kinetics of tissue stem cells; their dividing progeny cells; terminal non-dividing mature lineage cells produced; the number of cells transferred at each passage; and the length of the culture period between passages (See Additional Materials Table S1).

\subsection{Integration of Cell Culture Data and Computer Simulation to Develop a Method for Counting Adult Tissue Stem Cells}

Based on the cellular model in Figure 1, we developed software that simulates cumulative population doubling (CPD) data computed for serially passaged cultures based on using inputs for both measured culture factors (i.e., starting viable cell number, passage interval length, number of cells passaged, general cell viability, maximum cell number achievable) and unknown cell kinetics factors like stem cell fraction (See Additional Materials Table S1 for complete list; Figure S1 and Figure S2). The software program also includes probabilistic variance factors for all input factors. Some variances were known from experiment (e.g., the coefficient of variation [COV] for transient amplifying cell generation time (22\%) was determined by time-lapse microscopy); but most were assumed to fall within the typical range for cell culture and cell counting experimental errors (COV $=5-10 \%)$. 
Given these initial input factors, the Probabilistic Stem Cell Kinetics (PSCK) software computes families of CPD curves within specified, biologically relevant probabilistic ranges. From these simulations (Figure 2B), the PSCK software can delineate quantitative changes in the specific cell kinetics properties of the different cell types (See Figure 1) over the course of serial passaging, including in particular those of tissue stem cells. Figure 3 provides an example of this powerful computational feature of the method. Based on the differences in their cell kinetics features, all of the different cell types evolving in complex primary cell cultures can be delineated quantitatively. The computations in Figure 3, derived from the simulation in Figure 2, show the independent changes in the fractions of tissue stem cells, transiently amplifying progenitor cells, and terminally-arrested differentiated cells during serial culture. Further cell kinetics delineations are also possible, like asymmetrically self-renewing stem cells versus symmetrically self-renewing stem cells (data not shown).

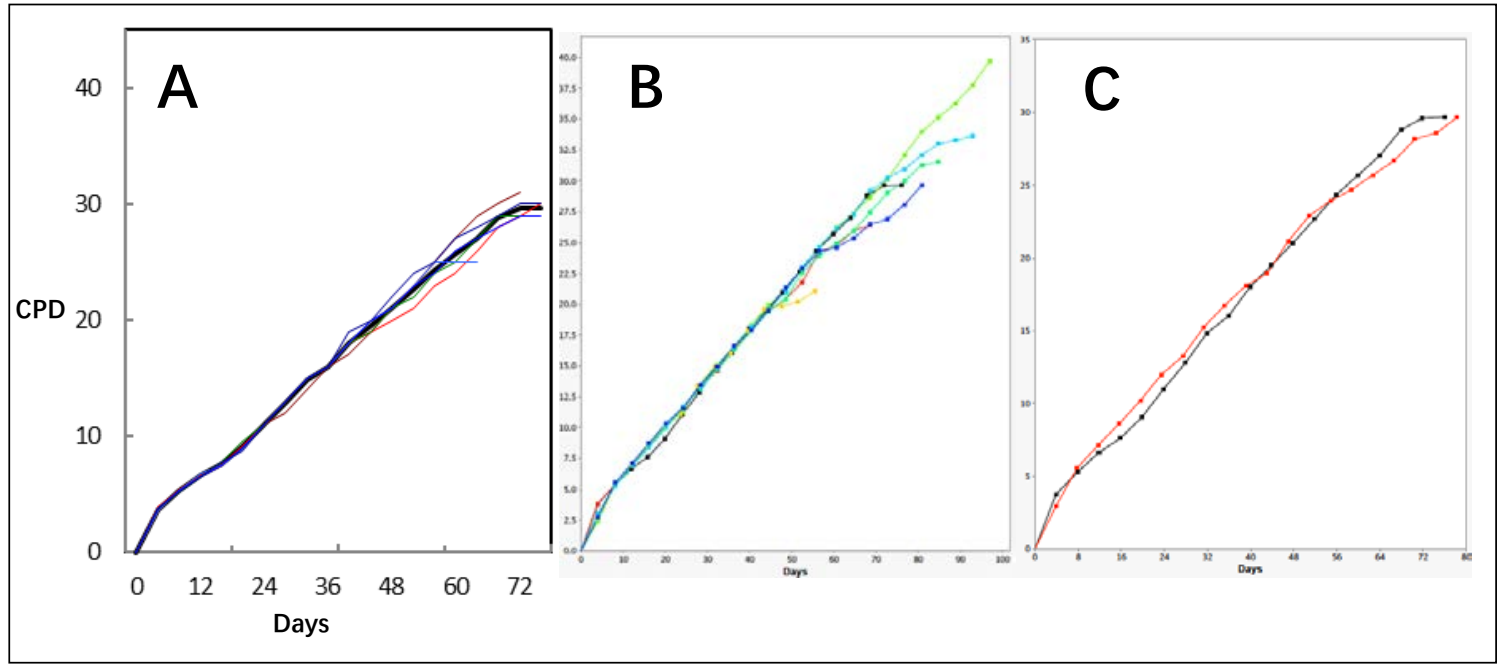

Figure 2 Example of PSCK simulation of serial culture cumulative population doubling data. A. Replicate (six) experimental CPD data from serial passage of pre-senescent human WI-38 lung fibroblasts. The black line traces the mean CPD values. B. PSCK simulation of WI-38 lung culture serial passage using input factors determined by RIFS computation. The black line is the mean CPD trace from the experimental data shown in A. C. Comparison of the PSCK-simulated mean CPD (red trace) to the experimental mean CPD data (black trace). The root mean squared error of the simulation compared to the experimental data was $0.19 \pm 0.11(p=0.024)$. 


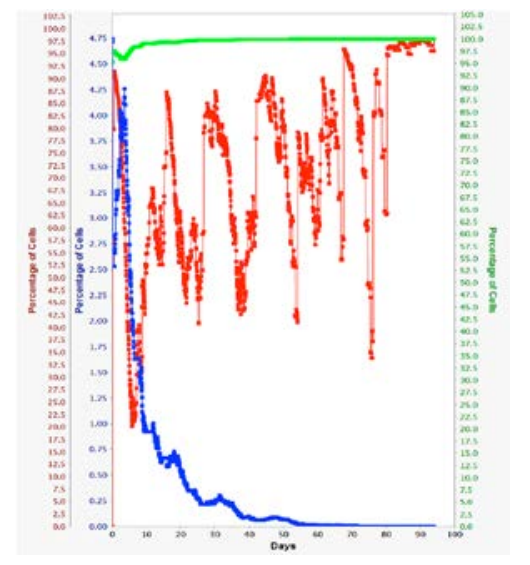

Figure 3 Examples of cell type-specific cell kinetics output from PSCK-RIFS computations. Shown are the computed outputs for the individualized cell kinetics of stem cells (NS, blue, middle y-axis), total transiently amplifying progenitor cells plus terminally-arrested differentiated cells (NT, green, right $y$-axis), and terminallyarrested differentiated cells alone (NT-Terminal, red, left $y$-axis) in terms of their percentage cellularity in serially passaged WI-38 cultures. Note that the difference between the green trace and the red trace corresponds to transiently amplifying committed progenitor cells specifically. Outputs were derived from the simulation data presented in Figure 2.

To discover the unknown input factors required for PSCK simulations, we developed a second integrated software program for Random Input Factor Searching (RIFS; See details in Additional Materials). The RIFS software simultaneously evaluates multiple, randomly-selected values for unknown input factors for the quality of PSCK simulations they yield compared to both the magnitude and the variance of replicate ( $n=3$ to 6 ) experimental CPD data. As shown in Figure 2 (Compare A to B), the RIFS program is able to discover input factor sets that produce CPD simulations that match experimental CPD with high statistical confidence. Importantly, the PSCK simulations not only achieve similar magnitude and variance, but they also show the cessation of cell division that is characteristic of primary mammalian cell cultures.

\subsection{Validations of the PSCK-RIFS Method for Counting Adult Tissue Stem Cells}

A first and essential validation of the new counting technology was its ability to simulate experimental CPD data with a high degree of statistical confidence (e.g., Figure 2B and 2C). We also used published serial cell culture data to extend the range of the different types of tissue stem cells evaluated. Although the published CPD data were generally from single serial cultures, the PSCK-RIFS analyses were sufficiently robust to yield results of high statistical confidence (See Table 1A).

Several different orthogonal analyses were used to validate the method further. The stem cells in all tissues examined to date were determined to decline in fraction with serial passaging (Figures 3 and 4; data not shown). This feature indicates that the commonly observed loss of stem cell functions with cell culture [24] can be accounted for by their asymmetric self-renewal kinetics as proposed previously [15]. After discovery of input factors to simulate published data for mouse embryo fibroblasts, we found that changes in only the input transferred cell number result in PSCK 
simulations that recapitulate the well-known cell density dependence of CPD curves for mouse embryo fibroblasts [18] (Figure 5). Previously, there had been no explanation for this effect. Our observation indicates that it can be readily explained by the intrinsic asymmetric cell kinetics properties of tissue stem cells.
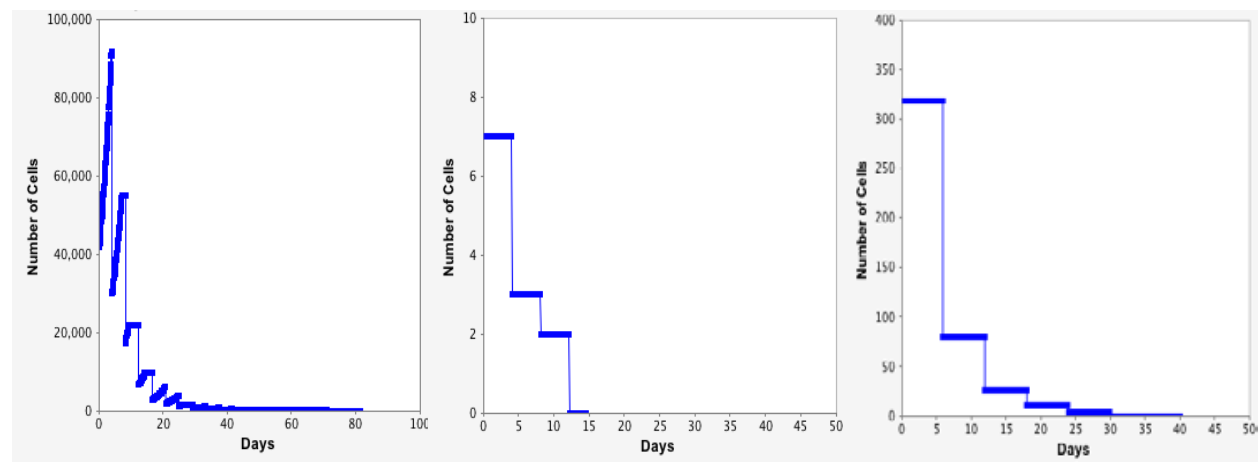

Figure 4 Use of PSCK-RIFS to compute the specific stem cell number and kinetics during the serial culture of cells derived from different human tissues. The cell kinetics factors determined from respective simulations like the example shown in Figure 2 were used to compute the number of tissue stem cells as a function of days of serial culture passage. The total number of viable cells input at the start of each culture $(0$ hour) was 325,000 (A, expanded liver stem cells [25]), 65,000 (B, HSCs in CD34+selected bone marrow cells), and 375,000 (C, MSCs from primary bone marrow [24]). Note the detection of symmetric self-renewal by liver stem cells ( $A$, inclined phases) between passages (perpendicular lines), but not for bone marrow-derived HSCs or MSCs ( $B$ and $C$, respectively, flat phases). This important biological distinction is quantified by the symmetric self-renewal rate, RS, (see Table 1B).
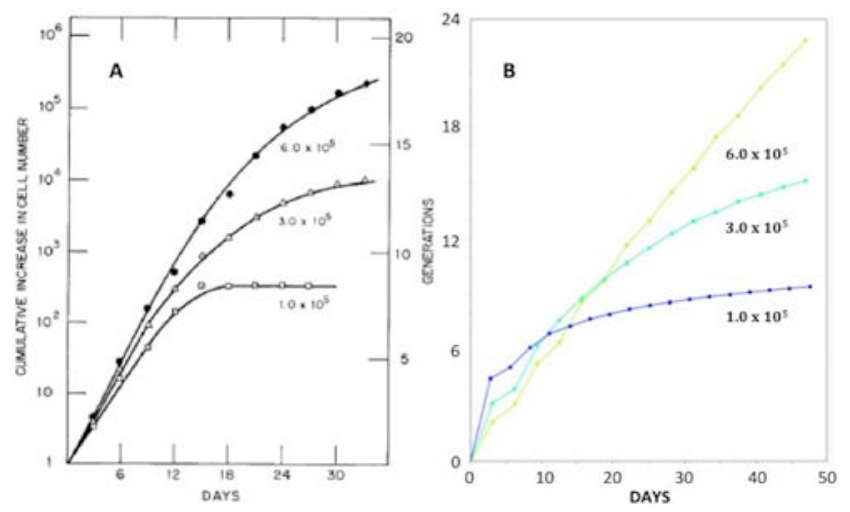

Figure 5 Ex vivo asymmetric self-renewal kinetics by tissue stem cells can explain the density dependence of serially passaged mouse embryo fibroblast cultures. A. Data reproduced with permission from Todaro and Green [18] (C1963 TODARO et al. Originally published in J. CELL BIOL. https://doi.org/10.1083/jcb.17.2.299) showing the increase in cumulative population doublings (generations) for serially passaged primary mouse embryo fibroblast cultures associated with the indicated increases in the number of cells transferred at 3 day intervals. B. After development of a PSCK simulation to approximate the data for the lowest transferred cell number $\left(1 \times 10^{5}\right.$ cells), the subsequent simulations were developed after changing only to the indicated input number for cells transferred in the simulation. 
Table 1 Quantitative validations of PSCK-RIFS for tissue stem cell counting and cell kinetics determinations.

A. Comparisons of PSCK-RIFS tissue stem cell fraction to reported functional determinations ${ }^{\mathrm{a}}$

\begin{tabular}{|c|c|c|c|c|c|c|c|}
\hline Tissue Type: & BM-CD34 $^{+}$ & CB-CD34+b,c & CB-CD34-b & Lung & Liver & MSC-Amniotic ${ }^{d}$ & MSC-BM $^{\mathrm{e}}$ \\
\hline PSCK-RIFS & $\begin{array}{l}2.6 \times 10^{-4} \\
\pm 5.5 \times 10^{-5 \ddagger}\end{array}$ & $\begin{array}{l}2.4 \times 10^{-3} \\
\pm 0.80^{-3 b \S} \\
0.08 \pm 0.06^{c *}\end{array}$ & $\begin{array}{l}1.2 \times 10^{-4} \\
\pm 0.0^{\ddagger \ddagger}\end{array}$ & $0.04 \pm 0.04^{* *}$ & $0.08 \pm 0.08^{* *}$ & $\begin{array}{l}3.1 \times 10^{-4} \\
\pm 1.4 \times 10^{-4 \neq}\end{array}$ & $\begin{array}{l}U^{f}-4.6 \times 10^{-4} \\
\pm 1.4 \times 10^{-4 \ddagger}\end{array}$ \\
\hline Functional & $6.2 \times 10^{-4}[26]$ & $\begin{array}{l}1.6 \times 10^{-3}[27], \\
0.025[28]\end{array}$ & $\begin{array}{l}\text { UD [29], } \\
4 \times 10^{-5}-0.001[28]\end{array}$ & $0.13[15]$ & $0.22 \pm 0.13[25]^{g}$ & N.A. ${ }^{h}$ & N.A. \\
\hline
\end{tabular}

${ }^{*}, p<0.05 ;{ }^{* *}, p<0.02 ; \neq, p<0.01 ; \neq \neq, p=0.001 ; \S, p<0.0001$

B. Detection of stem cell-specific cell kinetics effectors ${ }^{i}$

\begin{tabular}{|c|c|c|c|c|c|}
\hline Tissue/Agent & MSC-BM/Con ${ }^{\mathrm{e}}$ & $\mathrm{MSC}-\mathrm{BM} / \mathrm{hPL}^{\mathrm{e}}$ & 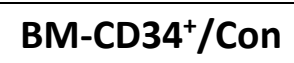 & BM-CD34 ${ }^{+} /$Xanthosine & $\mathrm{BM}-\mathrm{CD} 34^{+} / \mathrm{BCNU}$ \\
\hline \multicolumn{6}{|l|}{ Parameter } \\
\hline \multicolumn{6}{|l|}{ Stem Cells } \\
\hline Initial Fraction & $5.6 \times 10^{-4}(\mathrm{NS})^{\mathrm{j}}$ & $9.9 \times 10^{-4}(0.012)$ & $2.6 \times 10^{-4}(0.004)$ & $3.5 \times 10^{-3}(0.001)$ & $1.3 \times 10^{-4}(0.001)$ \\
\hline SSR Rate, RS & 0.040 (NS) & $5.2 \times 10^{-4}(\mathrm{NS})$ & $1.3 \times 10^{-3}(\mathrm{NS})$ & $3.2 \times 10^{-3}(0.037)$ & 0.0 (NS) \\
\hline Sym CC Time & $9.4 \mathrm{~h}(0.0033)$ & $11 \mathrm{~h}(\mathrm{NS})$ & $7.8 \mathrm{~h}(<0.0001)$ & $9.4 \mathrm{~h}(\mathrm{NS})$ & $8.2 \mathrm{~h}(\mathrm{NS})$ \\
\hline Asym CC Time & $16 \mathrm{~h}(\mathrm{NS})$ & $12 \mathrm{~h}(\mathrm{NS})$ & 7.0h (0.0002) & $6.6 \mathrm{~h}(\mathrm{NS})$ & 7.6 (NS) \\
\hline \multicolumn{6}{|c|}{ Committed Progenitor Cells } \\
\hline CC Time & $12 \mathrm{~h}(0.0034)$ & $9.8 \mathrm{~h}(\mathrm{NS})$ & $6.8 \mathrm{~h}(<0.0001)$ & $8.2 \mathrm{~h}(\mathrm{NS})$ & $6.4 \mathrm{~h}(\mathrm{NS})$ \\
\hline
\end{tabular}

$a$, values are the mean \pm standard deviation for $n=5$ determinations; $b$, data from ref. 30; $c$, data from ref. 31; d, data from ref. 32; e, data from ref. 24; $f$, UD, undetectable; $g$, data not shown; h, N.A., not available; i, values are the mean ( $p$ for different than control [Con] value) for $n=5$ determinations; $j, p$ for Con value different than $0.0 ; N S, p>0.05$. 
We independently evaluated four different human tissue cell preparations for which functional estimates of stem cell fraction were available. Functional estimates use measures of tissue cell repopulation or asymmetric self-renewal as indicators of tissue stem cells (See Table $1 \mathrm{~A}$ ). In all four cases - bone marrow-derived CD34+-selected cells, umbilical cord-derived CD34+-selected cells, pre-senescent lung cell strains, and expanded liver stem cell strains - the PSCK-RIFS determinations of stem cell fraction were similar to available values reported based on functional estimates.

The most important validations came from comparisons of PSCK-RIFS determinations of human HSC fractions to published HSC fraction data from limiting-dilution SCID mouse repopulating cell (LDSRC) assays. The LDSRC assay, though limited to estimation of only HSC fraction, gives the previously best available estimate of the HSC-specific fraction. The reported LDSRC assay value for human HSCs in CD34+-immunoselected bone marrow cell preparations is 6.2 per 10,000 total cells (Table 1A) [26]. Although the PSCK-RIFS determination of 2.6 is unequal to this value with statistical confidence $(p<0.0001$; Table $1 A)$, it is still quite similar numerically. The variance for the reported LDSRC assay determination was not available, making the statistical confidence determination for the difference potentially misleading. We identified two reports of LDSRC assay determinations of the HSC fraction in CD34+ fractions of human umbilical cord blood (Table $1 A$ ) $[27,28]$. The mean fraction of the reported values was $133 \pm 165$ per 10,000 total cells $(n=2)$, which was not statistically different than the mean of the two PSCK-RIFS analyses available using reported CPD data [30, 31] (Table 1A; $412 \pm 549 ; p=0.56$ ).

Human umbilical cord blood analyses also provided an independent validation of the specificity of PSCK-RIFS stem cell counts. Using published CPD data [30], the PSCK-RIFS analyses showed that HSCs fractionated, as predicted, preferentially to the CD34+ cell fraction in 20-fold excess over the CD34- cell fraction, in which HSCs were at the limit of detection for the analysis (Table $1 \mathrm{~A}$ ).

Additional validation came from evaluation of agents reported to alter tissue stem cell proliferation either positively or negatively. As shown in Table 1B, the new counting technology detected increases in the stem cell fractions of cultures supplemented with either xanthosine (Xs) or human platelet lysate (hPL). Xs is known to increase the frequency of symmetric stem cell divisions [17]; and hPL has been reported to increase the retention of mesenchymal stem cell (MSC) functions in culture [24].

Consistent with its previously described effects on tissue stem cells [17], Xs increased RS, the rate of symmetric self-renewal (See Additional Materials Table S1; Figure S2), for HSCs. The observed Xs-induced increase in HSC number was associated with an increase in RS from undetectable to $0.32 \%$ ( $p=0.037$ ) of HSC divisions. This small change in HSC symmetric selfrenewal kinetics is associated with a 10-fold increase in HSC number after only 4 days of culture. The two independently detected increases (RS and HSC number) are mathematically consistent. If only 3 out of 1000 HSCs (0.30\%) switched to symmetric self-renewal with an 8 hour generation time, in 96 hours, 12,288 (i.e., 10-fold) additional HSCs would be produced. hPL, which had a much smaller effect on human bone marrow-derived MSCs, did not show a significant effect on RS.

In contrast to the stem cell activation by $\mathrm{Xs}$ and $\mathrm{hPL}$, the chemotherapeutic agent carmustine, or bis-chloroethylnitrosourea (BCNU), caused a decrease in the determined HSC number within the first four days of culture. This agent has long been thought to be stem cell-toxic because it causes chronic organ failure, including chronic bone marrow failure [33]. 
As indicated by the data presented in Table 1, the PSCK-RIFS computational simulation technology can determine several important cell kinetics parameters of tissue stem cells, transiently amplifying lineage-committed cells, and terminally-arrested differentiated cells in complex cell preparations (See also Figure 3). Sub-routines of the program allow interrogation of intricate stem cell kinetics properties that have been previously inaccessible. We note the contrast between the cell kinetics of human lung or liver stem cells (Figure 4A, liver example) and human HSCs or MSCs (Figure 4B and 4C, respectively). The exponential inclines observed for human lung (data not shown) and liver stem cells (Figure 4A, liver) are due to symmetric self-renewal divisions that increase tissue stem cell number. These cell strains also had the highest observed RS values, $0.24 \pm 0.16(p<0.03)$ and $0.24 \pm 0.19(p<0.05)$, respectively, compared to CD34+ bone marrow cells, whose RS value was $0.0013 \pm 0.0011$ and not significantly different than 0.0 .

The high RS values signify that approximately $24 \%$ of stem cell divisions in these cultures are symmetric self-renewing divisions, which increase stem cell number. The exponential stem cell accumulation inclines (See Figure 4A) are another manifestation of these self-duplicating stem cell divisions. Within the cell kinetics model postulated, such a high rate of symmetric stem cell division is predicted to delay the rate of stem cell dilution, which would prolong the replicative span of the cultures. Consistent with this prediction, when initiated with the same number of total viable cells $(65,000)$ and passaged on the same schedule $(1 / 3$ culture splits every 96 hours; See Materials and Methods), cultures of CD34+-selected bone marrow cells reached division arrest much sooner (12 days) than cultures of lung cell strains or liver stem cell-enriched strains (60-76 days or 60 days, respectively). In contrast, consistent with their smaller RS values, bone marrowderived human HSCs and MSCs show only rare symmetric self-renewal divisions in culture. This property can explain the characteristic rapid disappearance of bone marrow-derived HSCs and MSCs in serial cell culture.

\subsection{Discovery of Algorithms for Determining the Initial Stem Cell Fraction from Simple Culture Population Doubling Time Data}

Using data outputs from PSCK-RIFS analyses, we were able to discover simple mathematical algorithms for convenient and rapid determination of the stem cell-specific fraction of tissue cell preparations. Because of the fundamental cell kinetics relationships described in Figure 1, the proliferation rate of any normal human tissue culture is related to the number of stem cells it contains, its stem cell fraction (SCF; FR_NS in Figure S2, Additional Materials). A universal measure of cell culture proliferation rate is the population doubling time (PDT). PDT is the time required for a cell culture to double in total cell number. Based on these ideas, we reasoned that it should be possible to deduce the number of tissue stem cells in any culture from the culture's PDT, which can be determined by simple counting of the total cells in the culture before and after a short period of culture.

A unique output of PSCK-RIFS analyses, the SCF over time (e.g., See Figure 3, blue line), makes it possible to define mathematical algorithms that yield the SCF from a culture's simple PDT. This discovery and its development are illustrated in Figure 6. The PSCK-RIFS software gives the two required data outputs. The first is the unique SCF throughout a foundational PSCK-RIFS serial culture analysis (Figure 6A; See also Figure 3, blue trace). Figure 6B shows an example of the second output, the corresponding data for the total culture cell number versus days of serial 
culture. The perpendicular declines in the data are simulations of culture dilutions to start next cultures in the series. The curvilinear inclines from dilution low points reflect the simulated proliferation of the diluted cells. The PDT between any two time points of culture can be calculated with Equation 2:

\section{Eq 2 PDT $=\left[\left(t_{2}-t_{1}\right) / \ln \left(N_{2} / N_{1}\right)\right] \ln 2$, where...}

$t_{1}=$ the starting time, $t_{2}=$ final time, $N_{1}=$ the initial cell number, $N_{2}=$ the final cell number

Computation of the PDT determined from time $=0$ of serial culture to the first maximum and for each incline in cell number from each successive dilution low point to the next maximum in Figure 6B can be performed and related to the corresponding SCF values at time $=0$ and at each culture dilution low point. These data are shown in Figure $6 \mathrm{C}$ and $6 \mathrm{E}$, respectively, for two different types of tissue stem cells. Both data plots show the predicted characteristic increasing culture PDT as the SCF decreases. The detection of this relationship is an orthogonal validation of the key principle upon with the PSCK-RIFS method is based, namely that tissue stem cells are ratedetermining for culture proliferative rate. As shown in Figure 6D and 6F, based on these analyses, mathematical conversions that linearize the PDT vs. SCF data into statistically significant descriptions of the data are readily found. Similar analyses, based on PDT values calculated with the experimental cell count data for the simulations, yield similar results (data not shown). These transforming PDT: SCF tissue stem cell counting algorithms make it possible to calculate confident estimates of the SCF of the same types of stem cells, when grown under the same culture conditions, from the PDT determined after 72 hours, and potentially less, of cell culture.

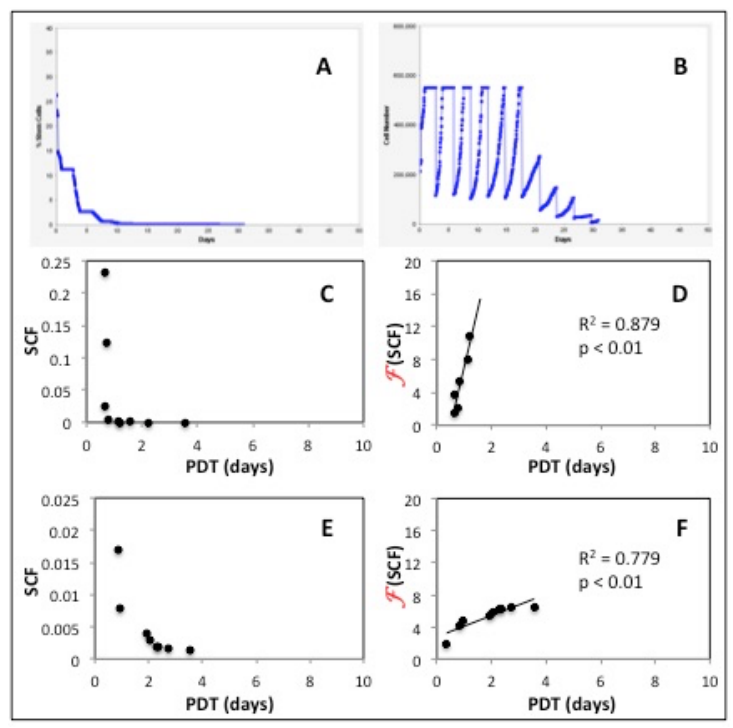

Figure 6 Defining population doubling time (PDT): stem cell fraction (SCF) algorithms. Examples of the SCF output ( $A$; $y$-axis, \% stem cells; $x$-axis, days) and total cell number output ( $\mathrm{B}$; $\mathrm{y}$-axis, cell number; $\mathrm{x}$-axis, days) for $\mathrm{CD}^{+} 4^{+}$cord blood cells. $\mathrm{C}$ and $\mathrm{E}$, examples of the derived plots of SCF versus PDT determined from corresponding total cell number data of $\mathrm{CD}_{3} 4^{+}$cord blood cells (derived from data in $\mathrm{A}$ and $\mathrm{B}$ ) and presenescent lung WI-38 cells (derived from data in Figure 2), respectively. D and F, mathematical linearization of the data in $C$ and $E$, respectively, to determine algorithms $(\mathcal{F})$ that define mathematical relationships between PDT and SCF 


\section{Discussion}

We anticipate that the ability to determine tissue stem cell specific-dose using the PSCK-RIFS technology and PDT: SCF algorithms will be of significant value for stem cell transplantation medicine. Past success in stem cell medicine has been primarily in the area of HSC transplantation medicine. Yet, even with HSC transplantation, there is still opportunity for significant improvements in efficacy that would occur with a means to determine HSC-specific dose. A prime example is umbilical cord blood HSC transplantation therapies, for which units fail at a high rate (18-24\%), because of insufficient stem cells [34]. Without being able to count HSCs, it has been impossible to know which cord blood units would have insufficient potency. This lack of information is a source of significant morbidity and deaths that could be avoided, if there were a method to routinely identify units with an adequate HSC dose. Transplants with adult bone marrow and mobilized peripheral blood are more reliable, with failure rates of a few percent [35]. However, this lower rate of failure may indicate that often patients receive excess HSCs. Being able to count HSCs would allow identification of scarce donor units that could be used to treat additional patients.

Since the earliest days of tissue stem cell research, mathematical and computer modelling have been employed as tools to investigate the biological properties of tissue stem cells with respect to their earliest progeny, lineage-specific committed progenitor cells [36, 37]. In particular, these approaches were applied to several challenging problems in stem cell biology that were difficult to interrogate by observational or experimental approaches. These more challenging problems included identifying and quantifying tissue stem cells in vivo, discovering the mathematical form of their competing self-renewal and production of committed progenitor cells, and investigating how tissue stem cells and their subtended cellular systems respond to physiological factors and exogenous agents like environmental toxicants and drugs [38-46]. Beyond relevance to important health-related processes in the body (e.g., normal growth and maturation, diseases like cancer, and aging), knowledge of such tissue stem cell properties is crucial to in vitro processes, too, like tissue engineering, stem cell manufacturing, stem cell transplantation medicine, and drug discovery.

The most actively pursued tissue stem cell modelling has focused on the identity and selfrenewal properties of hematopoietic stem cells [36, 38-42] or gastrointestinal tract stem cells, including primarily small intestinal epithelial stem cells $[12,47-49]$ and, to a lesser extent, colonic epithelial stem cells [43]. Recent modelling reports [40-52] focus on the still unsettled question whether tissue stem cells achieve their balance of self-renewal versus tissue cell renewal by stochastic or deterministic division and differentiation programs [41, 53-55]. The mouse has been the major experimental system for evaluating the validity of previously developed mathematical and computer models [41, 42]. As a result, only a dearth of human tissue stem cell modelling studies is available to date. Moreover, the majority of mouse studies are focused on interrogating in vivo processes that may not translate well to meet in vitro needs for improving progress in stem cell medicine.

The presented PSCK-RIFS method is grounded on the foundation of tissue cell kinetics concepts from earlier tissue stem cell modelling $[12,13,15]$ and experimental observations of human tissue cell strains maintained in serial cultures $[11,56,57]$. The experimental passaging schedules for the PSCK-RIFS approach differ in a fundamental way from those in foundational studies. The earlier 
studies were focused on maintaining culture proliferation for as long as possible; so that often cultures were held at confluence for extended periods. In marked contrast, cultures for PSCK-RIFS analyses are maintained on serial passage schedules that are designed for rapid declines in cell number with passaging, with cultures achieving a confluent monolayer state for only limited periods early in the serial culture, if ever at all. This strategy accelerates the decline in tissue stem cell number, which is the crucial effect that enables determination of tissue stem cell fraction and specific cell kinetics.

Unlike previous stem cell modelling approaches, whether mathematical or computational, the PSCK-RIFS method was expressly designed to discover previously inaccessible properties of cultured perinatal and postnatal tissue stem cells that constituted crucial gaps in knowledge that limited progress in stem cell research and stem cell medicine. The most significant among these knowledge gaps was a means to determine the tissue stem cell-specific fraction of complex tissue cell preparations, whether experimental samples, manufactured stem cell production lots, or therapeutic treatments. The PSCK-RIFS method can achieve this determination for any cell preparation that will proliferate in culture. Because the initial stem cell fraction is independent of subsequent culture conditions (and changes in the stem cell fraction during culture can be quantified), differences in culture media do not alter the initial stem cell fraction determination.

In contrast, the PDT: SCF stem cell counting algorithms developed with the output data of foundational PSCK-RIFS analyses are predicted to be very dependent on the culture conditions under which they were derived. Foundational PSCK-RIFS serial culture analyses discover tissue stem cell factors in the instant conditions, whatever they may be. However, the subsequently derived PDT: SCF algorithms are likely to often only apply to the same type of stem cell preparations under the same culture conditions as those of the foundational analysis. It is already evident that different stem cell types grown under different culture conditions have different PDT: SCF algorithms (Compare Figure 6D to Figure 6F). Even the same types of stem cells are predicted to have different PDT: SCF algorithms, if differences in culture conditions significantly alter either their cell kinetics properties of those of other cell types in the culture.

The advantages of the convenience and speed of PDT: SCF algorithms more than outweigh their requirement of specific culture conditions. For the same types of tissue stem cells under the same culture conditions, PDT: SCF algorithms enable timely stem cell counting on the timescale of days or less. Such a capability makes stem cell counting practical for use for laboratory tissue stem cell research, for optimizing and monitoring human cell biomanufacturing, for drug development assays, and for stem cell treatment dose determination.

A major challenge in the development of the PSCK-RIFS technology has been validating its specificity and accuracy. Although the presented validations are sufficient for this stage of development, we anticipate that continued use of the technology in other contexts will be important for its continued validation and improvement. For validation, we applied five different orthogonal approaches. The analyses utilized cumulative population doubling data from our own original cell culture analyses ( 5 analyses) as well as data from published serial culture analyses ( 8 analyses; [18, 24, 30-32]). The use of published cell count data reduced bias in the validations and demonstrated the versatility and facility of the method. For primary validation, we compared PSCK-RIFS determinations to literature reports of the HSC fraction in CD34+-selected human bone marrow cells and umbilical cord blood cells determined by LDSRC assays. The LDSRC assay is the most specific and best available method for estimating the fraction of any tissue stem cell type. 
The other four validation bases included comparison to independent determinations of the asymmetrically cycling cell fraction, which is the primary PSCK-RIFS basis for stem cells; analysis of differences in the PSCK-RIFS determination related to tissue stem cell fractionation (CD34 fractionation); analysis of changes in the PSCK-RIFS determination related to supplementation of agents either known (xanthosine) or suspected (hPL) of increasing tissue stem cell proliferation; and analysis of changes in the PSCK-RIFS determination after treatments with agents suspected of toxicity against tissue stem cells (BCNU).

We emphasize here that the basis for the validation of the PSCK-RIFS method and the derived PDT: SCF algorithms is not the use of any pre-existing molecular biomarkers, which are not specific for tissue stem cells. Our independent experimental quantification of HSCs in bone marrowderived CD34+-selected populations (Table $1 \mathrm{~A}$ ) is compared to LDSRC assays in the published literature. Similar validation comparisons, based on PSCK-RIFS determinations using published serial culture data, evaluated changes in HSC number associated with CD34+ fractionation of umbilical cord blood cells (Table 1A). None of these validations were based on CD34+ cell counts per se. All other validations used PSCK-RIFS determinations based on serial culture experiments performed in the present study. These included comparisons to independent determinations of asymmetrically self-renewing cells, which is the tissue stem cell-specific cell kinetics factor that is the basis for the PSCK model (Table 1A; lung stem cells and liver stem cells). Other validations based on original serial culture data evaluated predicted changes in the determined values of HSCs or MSCs in response to supplementation of cultures with either a positive effector of tissue stem cell self-renewal (Table 1B; xanthosine for HSCs) or a tissue stem cell-toxic agent (Table 1B; BCNU for HSCs).

These many different independent validations provide a high degree of confidence that the PSCK-RIFS method can provide a faithful estimation of the tissue stem cell-specific fraction of human cell preparations from a variety of source tissues. However, like any biological modelbased computational method, the accuracy and precision of determinations depend on the how well the underlying model defines the essential features of the evaluated biological system and the quality of the input data for computation. The PSCK model (Figure 1) has other input factors, in addition to the main ones that are measured experimentally or discovered by the RIFS software, that are used for the PSCK simulations and the RIFS software search (Additional Materials, Table S1). The additional factors, as noted in the main text, are unknown variances that are currently prescribed to fall within a limited "biological range." We have conducted sensitivity analyses to identify the factors that are the more significant determinants in PSCK simulations. However, we cannot exclude the possibility that the input variance assumptions may sometimes be incorrect and impact the accuracy of PSCK-RIFS determinations. Of course, to the extent that any significant feature of the model is incorrect, the accuracy and, potentially, the precision of stem cell fraction determinations will be affected. As the first general method for quantifying tissue stem cells specifically, the best future test of the PSCK-RIFS method will be the collective outcomes of its widespread use for investigation of applications in stem cell science and stem cell medicine. For example, the finding that the stem cell dose determinations by the PSCK-RIFS method are predictive of clinical outcomes in stem cell transplantation medicine would be an empirical validation of its value and ability to provide reliable tissue stem cell quantitation.

Beyond quantitative validation that a variety of tissue stem cells can be counted specifically, we also evaluated the statistical confidence of the accuracy of PSCK-RIFS determinations. Indicative of 
the statistically significant $p$-values for determinations in Table 1, the $95 \%$ confidence intervals provided in Table 2 of PSCK-RIFS stem cell fraction determinations over a diverse range of tissue stem cell types - show a high degree of resolution. A similar degree of statistical confidence is observed for other cell kinetics factors determined by the PSCK-RIFS method. The narrow range of the confidence intervals reveals that the five 1000-cycle RIFS search analyses (See Additional Materials), used to discover the stem cell fraction and other cell kinetics factors, yield a unique and single solution within the experimental error of the analysis. This quantitative characteristic is another indication of the quality of the PSCK-RIFS method.

Table 2 Analyses of the confidence intervals of PSCK-RIFS tissue stem cell fraction determinations ${ }^{1}$.

\begin{tabular}{lll}
\hline Tissue Type & $\begin{array}{l}\text { Tissue Stem Cell Fraction } \\
\text { (per 10,000 total cells) }\end{array}$ & 95\% Confidence Interval \\
\hline BM-CD34 $^{+}$ & 2.6 & $2.0-3.2$ \\
CB-CD34 $^{+}$ & 24 & $18-30$ \\
Lung & 420 & $120-720$ \\
Liver & 812 & $200-1420$ \\
MSC-Amniotic & 3.1 & $1.4-4.8$ \\
MSC-BM & 4.6 & $2.9-6.3$ \\
\hline
\end{tabular}

${ }^{1}$ Same determinations as in Table $1 \mathrm{~A}$.

In summary, the PSCK-RIFS technology and derivative PDT: SCF algorithms are a solution to the long-standing challenge of specific and accurate quantification of adult tissue stem cells. The technologies can be applied to tissue stem cells in complex cell preparations, including stem cell experiments, stem cell production cultures, and stem cell treatments. Adoption of the new method will accelerate progress in stem cell science and stem cell medicine by addressing longstanding unmet needs like determining the dose and quality of therapeutic stem cells. The technology can also be applied for evaluation of the effects of varied compounds on tissue stem cells and for optimizing the production of stem cells for cell therapy. Finally, the PSCK-RIFS technology is a new tool for evaluation of new classes of molecular biomarkers with potential to specifically identify and quantify adult tissue stem cells directly [3].

\section{Conclusions}

A first method for specific and accurate counting of perinatal and postnatal tissue stem cells is described. The method uses computer simulation of a probabilistic stem cell kinetics model - of how total cells are produced from tissue stem cells in culture - to discover previously inaccessible tissue stem cell kinetics factors, including among them the stem cell-specific fraction. The ability of the described Probabilistic Stem Cell Kinetics-Random Input Factor Searching (PSCK-RIFS) software to determine the stem cell-specific fraction of a variety of different human tissue cell preparations was validated by several different comparisons to the best available estimates of stem cell-specific fraction. These comparisons included estimates of human hematopoietic stem cell fraction by limiting-dilution SCID mouse repopulating cell assays and effects of tissue stem cell-active agents. A major advance enabled by PSCK-RIFS analyses was the discovery of 
Population Doubling Time: Stem Cell Fraction (PDT: SCF) algorithms that allow convenient and rapid determination of tissue stem cell fraction from simple culture population doubling time data. The new tissue stem cell counting technologies will enable stem cell-specific dose determination for stem cell transplantation medicine for the first time. Such capability is predicted to yield many advances in stem cell transplantation medicine, as well as tissue stem cell research, cell biomanufacturing, drug development, and environmental health science.

\section{Acknowledgments}

We thank Mr. Amin Nobari for assistance with evaluating the graphical user interface and the operation of the PSCK-RIFS software.

\section{Additional Materials}

The following additional materials are uploaded at the page of this paper.

1. Detailed description of PSCK-RIFS software operation.

2. Table S1: Probabilistic Stem Cell Kinetics (PSCK) Model Input Factors.

3. Figure S1: Generation of CPD data by serial culture.

4. Figure S2: Illustration of the stem cell asymmetric self-renewal culture model with inputs required for simulation of CPD data using the PSCK software.

5. Figure S3: RIFS software search analyses of PSCK factor sets.

\section{Author Contributions}

JLS conceived the PSCK-RIFS method and derivation of PDT: SCF algorithms, developed the PSCK computational model, devised the RIFS strategy, collaborated in the programming of the PSCK-RIFS software, performed all reported PSCK-RIFS and PDT: SCF algorithm analyses, and wrote the manuscript. RD collaborated in the programming of the PSCK-RIFS software and wrote the PSCK-RIFS computer code. LM collaborated in the initial PSCK computer programming. FA evaluated the quality of the PSCK-RIFS program and computer coding. RD, LM, and FA reviewed the manuscript.

\section{Funding}

Not applicable.

\section{Competing Interests}

The authors are either employed by companies or have financial interests in companies (AlphaSTAR Corporation and Asymmetrex LLC) that may gain or lose financially through this publication.

\section{References}

1. Ivanovic Z. Hematopoietic stem cells in research and clinical applications: The "CD34 issue." World J Stem Cells. 2010; 2: 18-23. 
2. Sherley JL. Principles for targeting adult tissue stem cells to achieve durable gene and geneediting therapeutics. Cambridge: Cambridge University Press; 2018. p. 285-300.

3. Sherley JL. Dose determination for stem cell medicine: A need whose time has come. Amsterdam: Elsevier; 2018. p. 383-397.

4. Caplan A. Mesenchymal stem cells: Time to change the name! Stem Cells Transl Med. 2017; 6: 1441-1445.

5. Sherley JL. Accelerating progress in regenerative medicine by advancing distributed stem cellbased normal human cell biomanufacturing. Pharm Anal Acta. 2014; 5: 286.

6. Rich IN. Improving quality and potency testing for umbilical cord blood: A new perspective. Stem Cells Transl Med. 2015; 4: 967-973.

7. Purton LE, Scadden DT. Limiting factors in murine hematopoietic stem cell assays. Cell Stem Cell. 2007; 1: 263-270.

8. Patterson J, Moore CH, Palser E, Hearn JC, Dumitru D, Harper HA, et al. Detecting primitive hematopoietic stem cells in total nucleated and mononuclear cell fractions from umbilical cord blood segments and units. J Transl Med. 2015; 13: 94.

9. Sherley JL. Asymmetric self-renewal: The mark of the adult stem cell. London: Imperial College Press; 2005. p. 21-28.

10. Sherley JL. New cancer diagnostics and therapeutics from a 9th "hallmark of cancer": Symmetric self-renewal by mutated distributed stem cells. Expert Rev Mol Diagn. 2013; 13: 797-810.

11. Hayflick L. The limited in vitro lifetime of human diploid cell stains. Exp Cell Res. 1965; 37: 614-636.

12. Potten CS, Morris RJ. Epithelial stem cells in vivo. J Cell Sci Suppl. 1988; 10: 45-62.

13. Loeffler M, Potten CS. Stem cells and cellular pedigrees-A conceptual introduction. San Diego: Harcourt Brace \& Co.; 1997. p.1-28.

14. Paré JF, Sherley JL. Biological principles for ex vivo adult stem cell expansion. San Diego: Elsevier, Inc.; 2006. p. 141-171.

15. Rambhatla L, Bohn SA, Stadler PB, Boyd JT, Coss RA, Sherley JL. Cellular senescence: Ex vivo p53-dependent asymmetric cell kinetics. J Biomed Biotech. 2001; 1: 27-36.

16. Merok JR, Sherley JL. Breaching the kinetic barrier to in vitro somatic stem cell propagation. J Biomed Biotech. 2001; 1: 24-26.

17. Lee HS, Crane GG, Merok JR, Tunstead JR, Hatch NL, Panchalingam K, et al. Clonal expansion of adult rat hepatic stem cell lines by suppression of asymmetric cell kinetics (SACK). Biotechnol Bioeng. 2003; 83: 760-771.

18. Todaro GJ, Green H. Quantitative studies of the growth of mouse embryo cells in culture and their development into established lines. J Cell Biol. 1963; 17: 299-313.

19. Majore I, Moretti P, Stahl F, Hass R, Kasper C. Growth and differentiation properties of mesenchymal stromal cell populations derived from whole human umbilical cord. Stem Cell Rev. 2011; 7: 17-31.

20. Duncan EL, Reddel RR. Genetic changes associated with immortalization. A review. Biochemistry (Mosc). 1997; 62: 1263-1274. 
21. Rogan EM, Bryan TM, Hukku B, Maclean K, Chang AC, Moy EL, et al. Alterations in p53 and p16INK4 expression and telomere length during spontaneous immortalization of Li-Fraumeni syndrome fibroblasts. Mol Cell Biol. 1995; 15: 4745-4753.

22. Harvey DJ, Levine AJ. P53 alteration is a common event in the spontaneous immortalization of primary BALB/c murine embryo fibroblasts. Genes Dev. 1991; 5: 2375-2385.

23. Sherley JL, Stadler PB, Johnson DR. Expression of the wild-type p53 antioncogene induces guanine nucleotide-dependent stem cell division kinetics. Proc Natl Acad Sci USA. 1995; 92: 136-140.

24. Heathman TR, Stolzing A, Fabian C, Rafiq QA, Coopman K, Nienow AW, et al. Scalability and process transfer of mesenchymal stromal cell production from monolayer to microcarrier culture using human platelet lysate. Cytotherapy. 2016; 18: 523-535.

25. Panchalingam K, Noh M, Huh YH, Sherley JL. Distributed stem cell kinetotoxicity: A new concept to account for the human carcinogenicity of non-genotoxic toxicants. London: Royal Society of Chemistry; 2016. p. 250-279.

26. Ziegler BL, Valtieri M, Porada GA, De Maria R, Müller R, Masella B, et al. KDR receptor: A key marker defining hematopoietic stem cells. Science. 1999; 285: 1553-1558.

27. De Wynter EA, Emmerson AJB, Testa NG. Properties of peripheral blood and cord blood stem cells. Baillieres Best Pract Res Clin Haematol. 1999; 12: 1-17.

28. Takahashi M, Matsuoka Y, Sumide K, Nakatsuka R, Fujioka T, Kohno H, et al. CD133 is a positive marker for a distinct class of primitive human cord blood-derived CD34-negative hematopoietic stem cells. Leukemia. 2014; 28: 1308-1315.

29. Kim DK, Fujiki Y, Fukushima T, Ema H, Shibuya A, Nakauchi H. Comparison of hematopoietic activities of human bone marrow and umbilical cord blood CD34 positive and negative cells. Stem Cells. 1999; 17; 286-294.

30. Engelhardt M. Telomerase regulation, cell cycle, and telomerase stability in primitive hematopoietic cells. Blood. 1997; 90: 182-193.

31. Durand B, Migliaccio G, Yee NS, Eddleman K, Huima-Byron T, Migliaccio AR, et al. Long-term generation of human mast cells in serum-free cultures of CD34+ cord blood cells stimulated with stem cell factor and interleukin-3. Blood. 1994; 84: 3667-3674.

32. Pratama G, Vaghjiani V, Tee JY, Liu YH, Chan J, Tan C, et al. Changes in culture expanded human amniotic epithelial cells: Implications for potential therapeutic applications. PLoS ONE. 2011; 6: e26136.

33. Marsh JC. The effects of cancer chemotherapeutic agents on normal hematopoietic precursor cells: A review. Cancer Res. 1976; 36: 1853-1882.

34. Olsson R, Remberger M, Schaffer M, Berggren DM, Svahn BM, Mattsson J, et al. Graft failure in the modern era of allogeneic hematopoietic SCT. Bone Marrow Transp. 2013; 48: 537-543.

35. Wolff SN. Second hematopoietic stem cell transplantation for the treatment of graft failure, graft rejection, or relapse after allogeneic transplantation. Bone Marrow Transpl. 2002; 29: 545-552.

36. Till JE, McCulloch EA, Siminovitch L. A stochastic model of stem cell proliferation, based on the growth of spleen colony-forming cells. Proc Natl Acad Sci USA. 1964; 5: 29-36.

37. Newton CM. Computer simulation of stem-cell kinetics. Bull Math Biophys. 1965; 27: 275-290.

38. Niewisch H, Vogel H, Matioli G. Concentration, quantitation, and identification of hemopoietic stem cells. Proc Natl Acad Sci USA. 1967; 58: 2261-2267. 
39. Nakahata T, Gross AJ, Ogawa M. A stochastic model of self-renewal and commitment to differentiation of the primitive hemopoietic stem cells in culture. J Cell Physiol. 1982; 113: 455-458.

40. Ema H, Takano $\mathrm{H}$, Sudo $\mathrm{K}$, Nakauchi $\mathrm{H}$. In vitro self-renewal division of hematopoietic stem cells. J Exp Med. 2000; 192: 1281-1288.

41. Roeder I, Kamminga LM, Braesel K, Dontje B, de Haan G, Loeffler M. Competitive clonal hematopoiesis in mouse chimeras explained by a stochastic model of stem cell organization. Blood. 2005; 105: 609-616.

42. Glauche I, Cross M, Loeffler M, Roeder I. Lineage specification of hematopoietic stem cells: Mathematical modeling and biological implications. Stem Cells. 2007; 25: 1791-1799.

43. Boman BM, Fields JZ, Bonham-Carter O, Runquist OA. Computer modeling implicates stem cell overproduction in colon cancer initiation. Cancer Res. 2001; 61: 8408-8411.

44. Potten CS, Booth C, Hargreaves D. The small intestine as a model for evaluating adult tissue stem cell drug targets. Cell Prolif. 2003; 36: 115-129.

45. Walters K. Colonic stem cell data are consistent with the immortal model of stem cell division under non-random strand segregation. Cell Prolif. 2009; 42: 339-347.

46. Barker $\mathrm{N}$, van Oudenaarden $\mathrm{A}$, Clevers $\mathrm{H}$. Identifying the stem cell of the intestinal crypt: Strategies and pitfalls. Cell Stem Cell. 2012; 11: 452-460.

47. Loeffler M, Birke A, Winton D, Potten C. Somatic mutation, monoclonality and stochastic models of stem cell organization in the intestinal crypt. J Theor Biol. 1993; 160: 471-491.

48. Podolsky DK. Regulation of intestinal epithelial proliferation: A few answers, many questions. Am J Physiol. 1993; 264: G179-G186.

49. Marshman E, Booth C, Potten CS. The intestinal epithelial stem cell. BioEssays. 2002; 24: 9198.

50. Snippert HJ, Van Der Flier LG, Sato T, Van Es JH, Van Den Born M, Kroon-Veenboer C, et al. Intestinal crypt homeostasis results from neutral competition between symmetrically dividing Lgr5 stem cells. Cell. 2010; 143: 134-144.

51. Simons BD, Clevers $\mathrm{H}$. Strategies for homeostatic stem cell self-renewal in adult tissues. Cell. 2011; 145: 851-862.

52. Klein AM, Simons BD. Universal patterns of stem cell fate in cycling adult tissues. Development. 2011; 138: 3103-3111.

53. Vogel H, Niewisch $\mathrm{H}$, Matioli G. The self-renewal probability of hemopoietic stem cells. J Cell Physiol. 1968; 72: 221-228.

54. Müller-Seiberg CE, Cho RH, Thoman M, Adkins B, Sieburg HB. Deterministic regulation of hematopoietic stem cell self-renewal and differentiation. Blood. 2002; 100: 1302-1309.

55. Vogel H, Niewisch H, Matioli G. Stochastic development of stem cells. J Theoret Biol. 1969; 22: 249-270.

56. Smith JR and Hayflick L. Variation in the life-span of clones derived from human diploid cell strains. J Cell Biology. 1974; 62: 48-53.

57. Macieira-Coelho A, Taboury F. A re-evaluation of the changes in proliferation in human fibroblasts during ageing in vitro. Cell Tissue Kinet. 1982; 15: 213-224. 


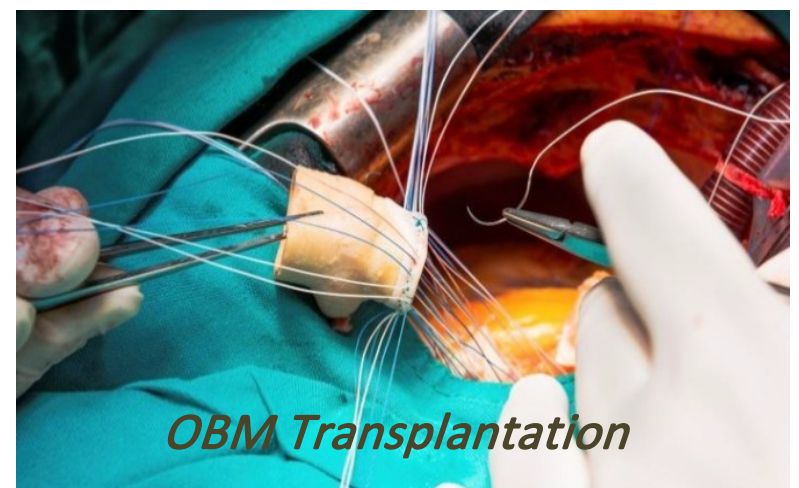

Enjoy OBM Transplantation by:

1. Submitting a manuscript

2. Joining in volunteer reviewer bank

3. Joining Editorial Board

4. Guest editing a special issue

For more details, please visit:

http://www.lidsen.com/journals/transplantation 